\title{
Fluorescence-Detected Two-Quantum and One-Quantum-Two-Quantum 2D Electronic Spectroscopy
}

\author{
Stefan Mueller, ${ }^{\dagger}$ Simon Draeger,${ }^{\dagger}$ Xiaonan Ma, ${ }^{\dagger}$ Matthias Hensen, ${ }^{\dagger}$ Tristan

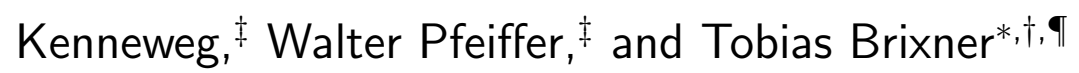

$\dagger$ Institut für Physikalische und Theoretische Chemie, Universität Würzburg, Am Hubland, 97074 Würzburg, Germany

$\ddagger$ Fakultät für Physik, Universität Bielefeld, Universitätsstr. 25, 33615 Bielefeld, Germany \Center for Nanosystems Chemistry (CNC), Universität Würzburg, Theodor-Boveri-Weg, 97074 Würzburg, Germany

E-mail: brixner@phys-chemie.uni-wuerzburg.de

\begin{abstract}
We demonstrate two-quantum (2Q) coherent two-dimensional (2D) electronic spectroscopy using a shot-to-shot-modulated pulse shaper and fluorescence detection. Broadband collinear excitation is realized with the supercontinuum output of an argon-filled hollow-core fiber enabling us to excite multiple transitions simultaneously in the visible range. The $2 \mathrm{Q}$ contribution is extracted via a three-pulse sequence with 16 -fold phase cycling and simulated employing cresyl violet as a model system. Furthermore, we report the first experimental realization of one-quantum-two-quantum (1Q-2Q) 2D spectroscopy, offering less congested spectra as compared to the $2 \mathrm{Q}$ implementation. We avoid scattering artifacts and nonreso-
\end{abstract}


nant solvent contributions by using fluorescence as the observable. This allows us to extract quantitative information about doubly excited states that agree with literature expectations. The high sensitivity and background-free nature of fluorescence detection allow for a general applicability of this method to many other systems.

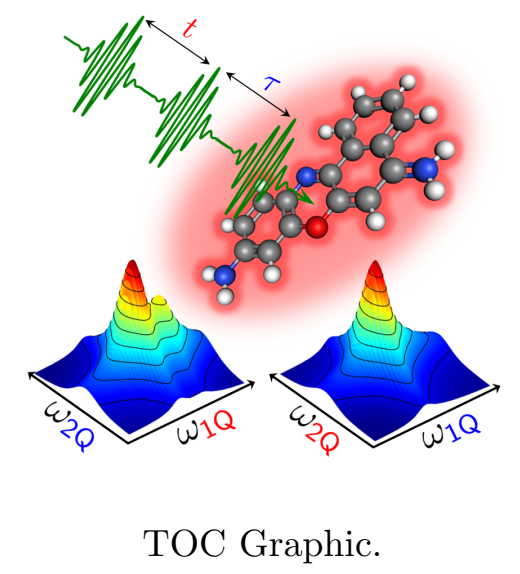

Over the past two decades, optical coherent two-dimensional (2D) spectroscopy ${ }^{1-4}$ has been applied in various fields, ranging from physical ${ }^{5,6}$ over chemical ${ }^{7-10}$ to biological science. ${ }^{11-16}$ By exciting superpositions of quantum states and probing their evolution via sequences of ultrashort phase-coherent laser pulses, detailed information about quantum dynamics can be revealed. After Fourier transformation of time-delay-dependent raw data, the signatures of correlated coherent dynamics of a system can be visualized as a 2D spectrum, identifying, e.g., electronic couplings and wave-packet dynamics.

Apart from well-established one-quantum (1Q) 2D spectroscopy, two-quantum (2Q) 2D spectroscopy has been carried out in 2004 by Zanni and coworkers for vibrational excitations in the infrared region ${ }^{17}$ and was proposed in 2007 by Mukamel and coworkers for electronic transitions as a means to quantify electron correlation energies. ${ }^{18}$ In $2 \mathrm{Q} 2 \mathrm{D}$ spectroscopy, a twice-interacting light field generates a 2Q coherence (also called double-quantum coherence) that is a superposition of the ground state with a two-photon allowed state, oscillating at a frequency in the range of twice the first $1 \mathrm{Q}$ transition frequency. With the follow- 
ing pulse of the excitation sequence this $2 \mathrm{Q}$ coherence is converted into its constituent $1 \mathrm{Q}$ coherences. Fourier transformation then leads to a 2D map of the frequencies of $2 \mathrm{Q}$ transitions directly correlated to those of the $1 \mathrm{Q}$ transitions. Thus, the core feature of this technique is the measurable shift of the energy correlation between two $1 \mathrm{Q}$ excitations. ${ }^{19}$ 2Q 2D spectroscopy was demonstrated on organic molecules, ${ }^{20,21}$ has been shown to be sensitive to molecular structure of organic chromophores, ${ }^{19,22}$ and also has been used to study many-body effects in quantum wells. ${ }^{23,24}$ Furthermore, it has proven to be useful for the determination of excited-state landscapes of biomolecules. ${ }^{25,26}$ To our knowledge, all 2Q 2D experiments were carried out in a noncollinear, coherent four-wave-mixing geometry utilizing laser pulses that are irradiated from three different directions toward the sample. In that approach, the desired signal is emitted in $\mathbf{k}_{1}+\mathbf{k}_{2}-\mathbf{k}_{3}$ direction, where $\mathbf{k}_{i}, i=1,2,3$, are the wave vectors of the three excitation laser pulses. This coherent signal is recorded via heterodyne detection with a local oscillator, yielding the nonlinear information in amplitude and phase. However, since there is no projection-slice theorem for $2 \mathrm{Q}$ signals, phasing of coherently detected $2 \mathrm{Q} 2 \mathrm{D}$ spectra has to be carried out via alternative procedures which can lead to erroneous results. ${ }^{21}$ Additionally, Gellen and coworkers previously showed that the comparatively strong nonresonant solvent response has to be taken into consideration. ${ }^{27}$ In fact, these circumstances can potentially exacerbate the evaluation of weak $2 \mathrm{Q}$ signals in box geometry.

Population-based 2D spectroscopy provides an alternative tool and has so far been applied successfully for $1 \mathrm{Q}$ spectroscopy. ${ }^{28-38}$ Here, the coherent information is encoded in the time delays and phases of the (collinear) excitation pulse sequence while detecting an incoherent signal. These incoherent signals can be photocurrents, ${ }^{30,33,36}$ or - as utilized in this work fluorescence. ${ }^{29,31,32,34,35,37,38}$

In the present work, we introduce fluorescence-detected 2Q 2D spectroscopy and demonstrate it on a model system (cresyl violet). Fluorescence is emitted from the quantum system after interaction with a three-pulse train as a function of pulse-specific phases $\phi_{i}$ and times 
a)

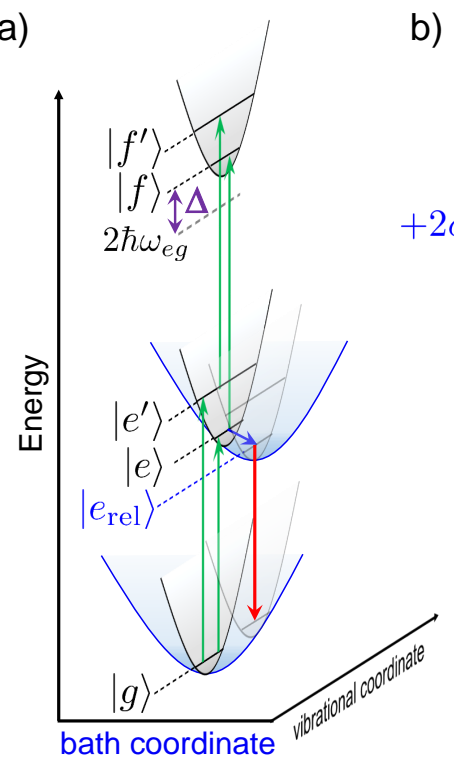

d)

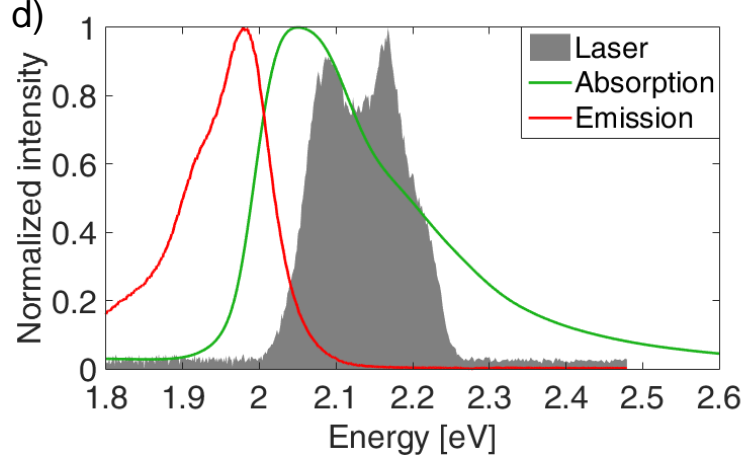

b) time $\uparrow$

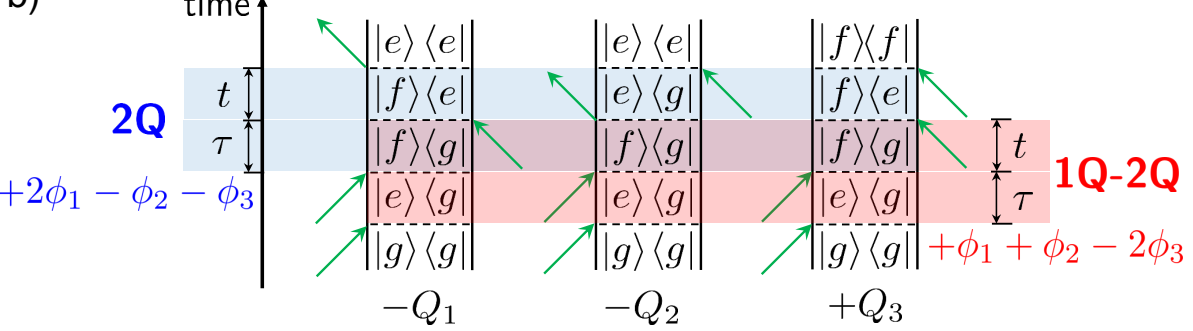

c) beam stabilization system argon-filled

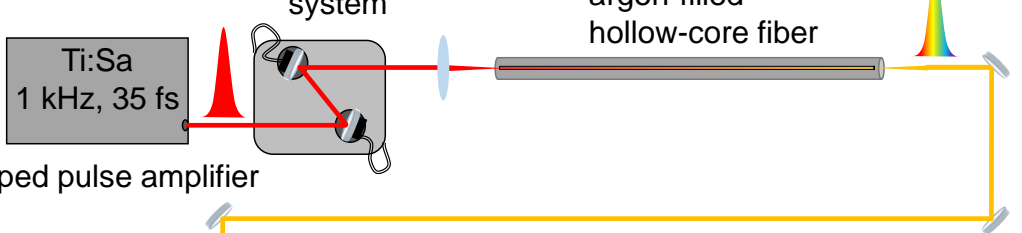

chirped pulse amplifier

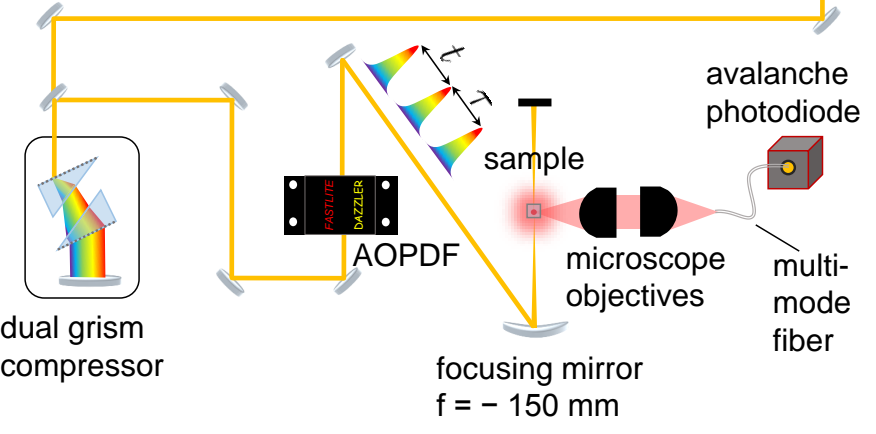

Figure 1: Fluorescence-detected 1Q, 2Q, and 1Q-2Q 2D spectroscopy. (a) Energy level scheme of a model system with electronic states $g, e, f$, vibrational sublevels indicated by primes, and $e_{\text {rel }}$ corresponding to the relaxed state from which fluorescence (red arrow) occurs after the inertial solvent response. Transitions via laser excitation are indicated by green arrows. The energy $\Delta=\hbar \omega_{f g}-2 \hbar \omega_{e g}$ is defined as the correlation energy. (b) Feynman diagrams for 2Q (blue) and 1Q-2Q (red) 2D spectroscopy. (c) Experimental setup: Long-term stable and broadband excitation is generated by an argon-filled hollow-core fiber. Multipulse sequences with defined time delays and phases are generated on a shot-to-shot basis by an acousto-optical programmable dispersive filter (AOPDF), and fluorescence is collected at a right angle. (d) Linear absorption (green) and fluorescence (red) of cresyl violet perchlorate $(0.1 \mathrm{mM}$ ) in ethanol (absorption maximum at $601 \mathrm{~nm}$, fluorescence maximum at $626 \mathrm{~nm}$ ). The excitation spectrum used for excitation (grey) is measured behind the AOPDF.

$t_{i}, i=1,2,3$, at which an interaction with a light field takes place. This necessitates that the last interaction transfers the system into an excited-state population from which fluorescence occurs directly, or into an energetically higher excited state which can subsequently decay nonradiatively into the fluorescent state. For this work, an electronic three-level monomer 
system consisting of a ground state $|g\rangle$, a singly excited state $|e\rangle$, and a 2Q state $|f\rangle$ with their respective vibrationally excited sublevels $\left|e^{\prime}\right\rangle$ and $\left|f^{\prime}\right\rangle$ shall be considered (Figure 1a). A vibrationally excited ground state is neglected here because such a state would not be involved in any 2Q Liouville pathway. An additional sublevel $\left|e_{\text {rel }}\right\rangle$ represents the excited state after solvent-shell relaxation and thus effectively incorporates the dynamic Stokes shift in a simplified manner, a process known to occur on the scale of few tens of femtoseconds in the employed solvent. ${ }^{39-41}$

If only coherences between the ground state and the singly excited state as well as between the singly and 2Q-excited states are generated in a 2D experiment, these coherence types are referred to as one-quantum (1Q) coherences, which can be rephasing or nonrephasing. ${ }^{4}$ For 2Q 2D spectroscopy we consider the coherent superposition of the ground state and the $2 \mathrm{Q}$ state generated via a two-photon process during a three-pulse sequence. The double-sided Feynman diagrams (Figure 1b, blue) display the relevant contributions (shown for three energy levels for simplicity) wherein we defined the $2 \mathrm{Q}$ coherence time $\tau$, which implies a double interaction of the first pulse with the phase $2 \phi_{1}$, and the $1 \mathrm{Q}$ coherence time delay $t$ between the following two pulses, interacting with the phases $-\phi_{2}$ and $-\phi_{3}$, respectively. ${ }^{42}$

From these pathways, the only positive-signed pathway $Q_{3}^{2 \mathrm{Q}}$ ends up in $|f\rangle$. Invoking Kasha's rule, ${ }^{43}$ fluorescence is only emitted via the first excited state $|e\rangle$. Hence, one needs to take into account that the fluorescence yield associated with a pathway that ends up in $|f\rangle$ may be less than unity due to nonradiative deactivation pathways. ${ }^{31,32,36}$ Note that, compared to coherence-detected $2 \mathrm{Q}$ spectroscopy, the additional pathway $Q_{3}^{2 \mathrm{Q}}$ interferes with $Q_{1}^{2 \mathrm{Q}}$ with opposite sign. Due to the negative signs for the pathways $Q_{1}^{2 \mathrm{Q}}$ and $Q_{2}^{2 \mathrm{Q}}$, one expects a strong negative signal in a real-valued $2 \mathrm{Q}$ spectrum. The involvement of these three pathways will not lead to a cancellation of the overall signal for identical 1Q transition frequencies as it would be the case in noncollinear phase-matched geometry with coherent detection. ${ }^{18}$ The energy shift between two consecutive $1 \mathrm{Q}$ transitions is defined as $\Delta=\hbar \omega_{f g}-2 \hbar \omega_{e g}$, where $\omega_{f g}$ is the transition frequency between the ground and the $2 \mathrm{Q}$ state 
and $\omega_{e g}$ the transition frequency between the ground and the first excited $1 \mathrm{Q}$ state. ${ }^{18,19,22}$ In our case, these frequencies can be extracted from a real-valued 2Q 2D spectrum via the position of the resulting peak with coordinates $\left(\hbar \omega_{\tau}, \hbar \omega_{t}\right)=\left(\hbar \omega_{f g}, \hbar \omega_{e g}\right)$, stemming from pathway $Q_{2}^{2 \mathrm{Q}}$ (Figure $\left.1 \mathrm{~b}\right)$.

Another type of nonlinear signal, which involves a $2 \mathrm{Q}$ coherence and is exclusively accessible via population-based $2 \mathrm{D}$ spectroscopy, is the so-called one-quantum-two-quantum (1Q-2Q) correlation contribution that has been suggested theoretically but not measured experimentally to our knowledge. ${ }^{42}$ This signal arises from an "inverted" $2 \mathrm{Q}$ experiment, where first a $1 \mathrm{Q}$ coherence is created which is then, after time $\tau$, subsequently converted into a $2 \mathrm{Q}$ coherence and probed after time delay $t$. The underlying phase combination is $\phi^{1 \mathrm{Q}-2 \mathrm{Q}}=\phi_{1}+\phi_{2}-2 \phi_{3}$ (Figure $\left.1 \mathrm{~b}, \mathrm{red}\right)$. The major advantage of $1 \mathrm{Q}-2 \mathrm{Q}$ versus conventional $2 \mathrm{Q}$ spectroscopy is that the $1 \mathrm{Q}-2 \mathrm{Q}$ pathways contain only the single $1 \mathrm{Q}$ coherence $|e\rangle\langle g|$ within the associated coherence time interval $\tau$, whereas $2 \mathrm{Q}$ spectroscopy probes the evolution of both $|f\rangle\langle e|$ and $|e\rangle\langle g|$ within the $1 \mathrm{Q}$ time interval $t$ (Figure 1b, blue). ${ }^{42}$ (Note that the roles of $\tau$ and $t$ are interchanged in the two methods.) As a result, the 1Q-2Q spectrum of a three-level system features only one resonance located at $\left(\hbar \omega_{\tau}, \omega_{t}\right)=\left(\hbar \omega_{e g}, \hbar \omega_{f g}\right)$ whereas the $2 \mathrm{Q}$ spectrum contains a superposition of two peaks located at $\left(\hbar \omega_{\tau}, \hbar \omega_{t}\right)=\left(\hbar \omega_{f g}, \hbar \omega_{e g}\right)$ and $\left(\hbar \omega_{\tau}, \hbar \omega_{t}\right)=\left(\hbar \omega_{f g}, \hbar \omega_{f e}\right)$, making the retrieval of the energy shift $\Delta$ more difficult if these peaks overlap (see Figure S1 in Supporting Information).

We use a 16 -fold $(1 \times 4 \times 4)$ phase-cycling scheme ${ }^{42}$ to recover the rephasing $1 \mathrm{Q}$ (not shown), 2Q, and 1Q-2Q contributions simultaneously from the same raw data set while measuring in a rotating-frame environment. In order to resolve $2 \mathrm{Q}$ coherences, the coherence time step sizes have to be shorter than in a 1Q 2D experiment according to the Nyquist limit. We here increment $\tau$ and $t$ from 0 to 90 fs in steps of 3 fs which is sufficient to recover all contributions. The schematic setup for broadband fluorescence-detected 1Q, 2Q, and 1Q-2Q 2D spectroscopy is depicted in Figure 1c and described in the Methods section.

For simulations, we solve numerically the Lindblad quantum master equation ${ }^{44}$ (see 
Methods and Supporting Information for details) for an electronic three-level system with additional vibronic sublevels (Figure 1a). In this simple model, we assume equal excitedstate vibrational frequencies. ${ }^{22}$ It is vital to describe the first singly and the doubly excited electronic state consisting of two vibrational sublevels each because organic dye molecules such as cresyl violet exhibit vibrational progression ${ }^{45}$ (see the shoulder in the linear absorption spectrum of Figure 1d) and because the vibronic structure has a major influence on the overall appearance of the 2Q 2D spectrum as has been shown by Kim and coworkers. ${ }^{19,22}$
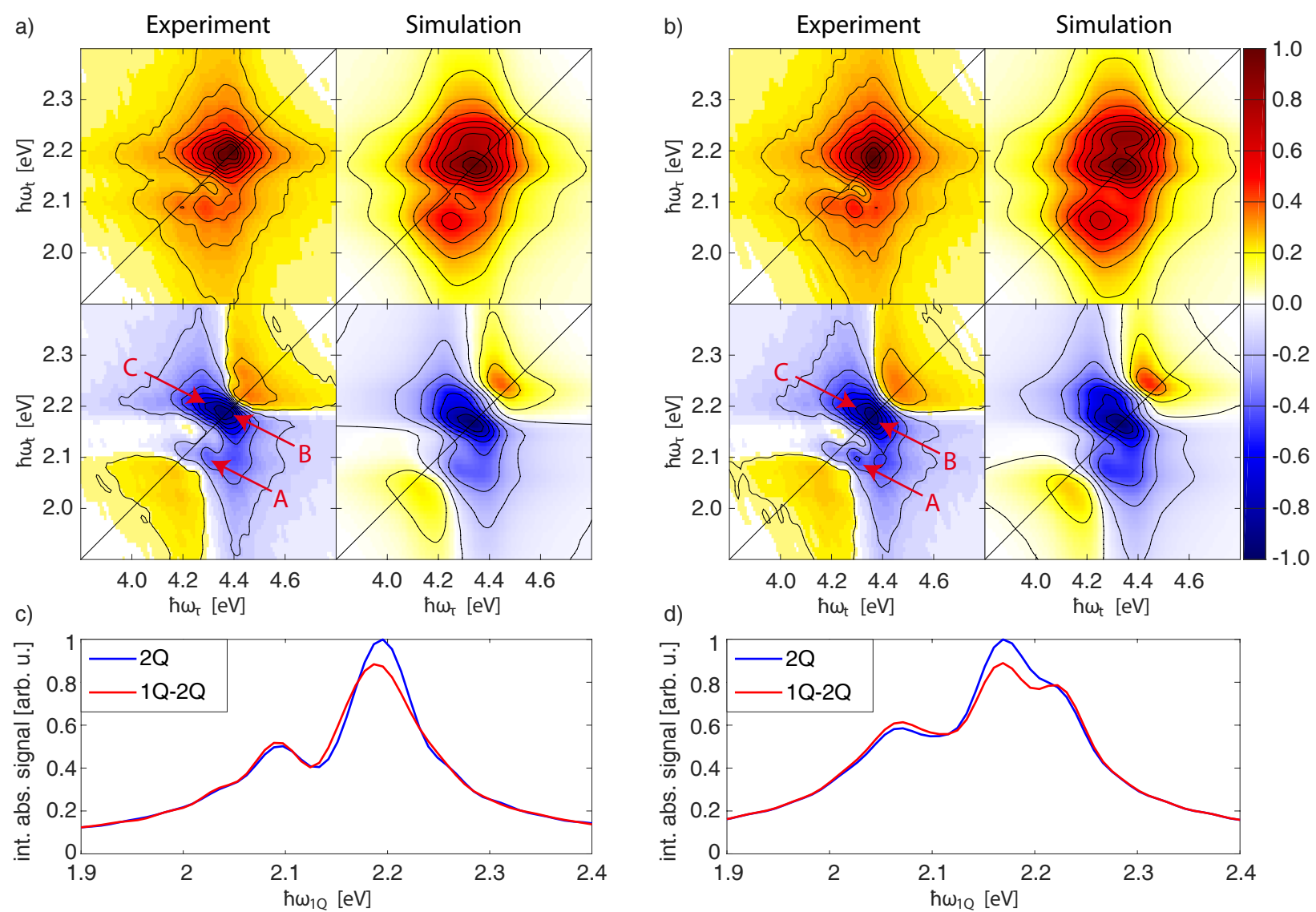

Figure 2: (a, b) Experimental and simulated 2Q 2D (a) and 1Q-2Q (b) spectra of cresyl violet in ethanol, both shown in absolute (top) and real values (bottom), normalized to the highest absolute value of each plot. The diagonal of the spectrum is marked with a line at $\hbar \omega_{\tau}=2 \hbar \omega_{t}$ (a) and $\hbar \omega_{t}=2 \hbar \omega_{\tau}$ (b). Distinct features (A-C) are marked in the real-valued spectra. All plots are drawn with nine contour lines. (c, d) Integrated 1Q projections of 2Q and 1Q-2Q spectra (both normalized to the same absolute value) from experimental (c) and simulated (d) absolute-valued data.

The experimental 2Q 2D spectra are depicted in Figure 2a, left, as absolute value (top) and real part (bottom). The simulated spectra are shown in Figure 2a, right, reproducing 
the measured spectra extremely well. Furthermore, it is evident that the signals are not accompanied with either scattering contributions or nonresonant response from the solvent that pose significant challenges in coherence-detected 2Q 2D spectroscopy. ${ }^{27}$ The absolutevalued spectrum shows two row-shaped features at $\hbar \omega_{t}=2.089 \mathrm{eV}$ and $\hbar \omega_{t}=2.195 \mathrm{eV}$, reflecting the existence of the two vibrational sublevels in the first electronic state $|e\rangle$ and $\left|e^{\prime}\right\rangle$, respectively. The high-energy peak has a larger amplitude than the low-energy peak, which seems to be contrary to expectations from the linear absorption profile where the relative heights are inverted. However, this behavior is reproduced by the simulations and can thus be attributed to a combined effect of the finite laser excitation bandwidth and the inertial solvent relaxation, by which $|e\rangle$ relaxes along a global bath coordinate immediately after photoexcitation towards the final emissive state $\left|e_{\text {rel }}\right\rangle$ (Figure 1a). ${ }^{40,41}$ To reproduce the experimental $2 \mathrm{Q}$ spectra accurately, we found a time constant of $10 \mathrm{fs}$ for the relaxation of $|e\rangle$ to $\left|e_{\text {rel }}\right\rangle$ to be best suited. The row-shaped peaks span a wider range along the $2 \mathrm{Q}$ axis $\hbar \omega_{\tau}$, indicating that the $2 \mathrm{Q}$ coherences dephase much faster than the $1 \mathrm{Q}$ coherences. As mentionend above, negative features dominate the real part of the $2 \mathrm{Q}$ spectrum so that the three negative-signed peaks A, B and C can be assigned to Liouville pathways in which the $2 \mathrm{Q}$ frequencies $\omega_{f g}$ (Peak A and C) and $\omega_{f^{\prime} g}$ (Peak B) are correlated to their corresponding 1Q frequencies. The overall orientation of the real-valued signal signifies the nonrephasing nature of the $2 \mathrm{Q}$ signal. The weaker positive features which appear besides the strong negative features in both the experimental and simulated real-valued spectra cannot be assigned to any quantum transfer pathway since they are part of the phase-twisted lineshapes themselves. ${ }^{21}$

The 1Q-2Q 2D spectrum can also be extracted from the same raw data. Note that the $2 \mathrm{Q}$ axis results from the first time delay, $\tau$, in $2 \mathrm{Q}$ spectroscopy (Figure 2a), and from the second time delay, $t$, in $1 \mathrm{Q}-2 \mathrm{Q}$ spectroscopy (Figure $2 \mathrm{~b}$ ). Thus we swap the two frequency axes in plotting the latter to have the $2 \mathrm{Q}$ frequency axis along the horizontal direction in both cases. It can be seen immediately that the two plots are very similar. Although theory 
states that a $1 \mathrm{Q}-2 \mathrm{Q}$ spectrum may contain less features along the $1 \mathrm{Q}$ axis compared to the 2Q spectrum, ${ }^{42}$ we find also two features along that axis. This happens when the energy shift of the $2 \mathrm{Q}$ state is comparable to the vibrational energy separation, as it was modeled in the simulation (see also Figure 1a). Then the only two distinguishable 1Q frequencies are $\omega_{e g}$ and $\omega_{e^{\prime} g} \approx \omega_{f e} \approx \omega_{f^{\prime} e^{\prime}}$. Furthermore we assume a partial cancellation of $Q_{1}$ and $Q_{3}$ pathways leading to a comparably dominant $Q_{2}$ pathway containing the $1 \mathrm{Q}$ coherences $|e\rangle\langle g|$ and $\left|e^{\prime}\right\rangle\langle g|$ in both spectra. This implies a high internal conversion efficiency from $|f\rangle$ into the fluorescent state $|e\rangle$. We can extract a signature of the pathways in which the time evolution of coherences between the 2Q-excited and the first singly excited states are probed by comparing projections onto the $1 \mathrm{Q}$ axis of the $2 \mathrm{Q}$ and $1 \mathrm{Q}-2 \mathrm{Q} 2 \mathrm{D}$ spectra in Figures $2 \mathrm{c}$ and $2 \mathrm{~d}$. We observe that the absolute-valued peak intensity of the high-energy region is higher in the case of $2 \mathrm{Q}$, which we attribute to the signal overlap at $\omega_{e^{\prime} g} \approx \omega_{f e} \approx \omega_{f^{\prime} e^{\prime}}$ in the $2 \mathrm{Q}$ spectrum. Nevertheless, the mentioned partial pathway cancellation diminishes this difference.

Assigning peak $\mathrm{A}$ in the experimental spectrum to pathway $Q_{2}^{2 \mathrm{Q}}$ in Figure 1a, we read off $\Delta_{\exp } \approx \hbar \omega_{f g}-2 \hbar \omega_{e g}=111 \mathrm{meV}$, which is in fair agreement with the value measured by Kim et al. (94 $\pm 5 \mathrm{meV})$ in a coherence-detected 2Q 2D experiment. ${ }^{19,22}$ The energy shift determined from peak $\mathrm{A}$ in the $1 \mathrm{Q}-2 \mathrm{Q}$ spectrum (Figure $2 \mathrm{~b}$ ) yields the same value. These results indicate that the same information about doubly excited states can be obtained in principle from both 2Q 2D and 1Q-2Q 2D spectroscopy, but for more complex systems 1Q2Q spectroscopy may be preferable since it avoids interference from the second electronic 1Q coherence (see also Section S1 and Figure S1 in Supporting Information). However, uncertainties in the determination of $\Delta$ may arise due to finite-pulse-duration effects ${ }^{46,47}$ and modulations of the spectral laser profile (see also Sections S1 and S2 in Supporting Information). We therefore utilized $\Delta_{\exp }$ as a starting parameter for a series of simulations with varying correlation energies. By comparing the simulated $2 \mathrm{Q}$ and $1 \mathrm{Q}-2 \mathrm{Q}$ spectra with the experimental data, we find that the smallest root-mean-square deviation is obtained for 
a value of $\Delta=121 \mathrm{meV}$, leading to the plots in Figure 2.

Summarizing, we presented the first experimental realization of fluorescence-based 2Q 2D spectroscopy and of electronic 1Q-2Q 2D spectroscopy. Using fluorescence as an observable removes contributions from the nonresonant solvent response and from scattering that can be significant issues in coherent four-wave-mixing detection. Furthermore, by using phase cycling in combination, ${ }^{28,29}$ an additional phasing procedure is obsolete. With phase cycling we also extracted various nonlinear contributions from one single data set. As an example, we obtained the energy offset between two 1Q excitations that comprise a two-photon allowed electronic state of cresyl violet via $2 \mathrm{Q}$ and $1 \mathrm{Q}-2 \mathrm{Q}$ spectral analysis in good agreement with literature values ${ }^{19,22}$ and determined the correlation energy by systematic simulations based on a simple six-level Lindblad master equation approach. We observed that in a molecular monomer like cresyl violet, overlapping features or pathway cancellation due to internal conversion into the first electronically excited state lead to no significant differences between 2Q and 1Q-2Q spectra, except for slightly varying peak amplitudes. However, we envision that $1 \mathrm{Q}-2 \mathrm{Q}$ spectroscopy may especially be useful in molecular aggregates and similarly complex systems for which the fluorescence quantum yields of pathways ending in different excited-state populations are not equal. In that case, 1Q-2Q spectra are less congested and lead to a higher level of confidence for quantitative analysis.

\section{Methods}

The experiment is based on the setup for $1 \mathrm{Q} 2 \mathrm{D}$ fluorescence spectroscopy that we reported earlier. ${ }^{38}$ In contrast to the former implementation, however, that employed a noncollinear optical parametric amplifier, we now use an argon-filled hollow-core fiber (HCF) for broadband continuum generation. ${ }^{48}$ A long-term stable continuum is ensured by utilizing an active beam stabilization system (Aligna, TEM Messtechnik GmbH). After dispersion pre-compensation by a dual grism compressor, we employ a commercial acousto-optical 
programmable dispersive filter (AOPDF, Dazzler, Fastlite) to select the spectral region of interest, see Figure 1d, and to switch between all required time delays and phases on a $1 \mathrm{kHz}$ shot-to-shot basis, enabling rapid data acquisition. ${ }^{38}$ Pulse-shaper-assisted collinear frequency-resolved optical gating $(\mathrm{cFROG})^{49}$ reveals compressed pulses with a duration of $<20$ fs (intensity FWHM). The collinear pulse trains are focused into the sample, which is circulated through a $(250 \mu \mathrm{m})^{2}$ cross-section fused-silica capillary-type flow cell (131.310-QS, Hellma), and the emitted fluorescence signal is collected at a right angle via microscope objectives and led to an avalanche photodiode (APD410x, Thorlabs). With this setup, the raw data containing various nonlinear signal contributions is acquired in 16 seconds. Averaging $(2000 \times)$ is performed for improvement of data quality. The sample consists of a $0.1 \mathrm{mM}$ solution of commercial cresyl violet perchlorate (Radiant Dyes $\mathrm{GmbH}$ ) in ethanol (analytical grade, Fisher Scientific UK) at ambient conditions.

For simulations we solve numerically, using a program package in MatLab R2017b, the Lindblad quantum master equation, ${ }^{44}$

$$
\frac{\partial}{\partial t} \rho\left(t^{\prime}\right)=-\frac{i}{\hbar}\left[H\left(t^{\prime}\right), \rho\left(t^{\prime}\right)\right]+\sum_{j} \frac{1}{T_{j}}\left(\mathcal{L}_{j} \rho\left(t^{\prime}\right) \mathcal{L}_{j}^{\dagger}-\frac{1}{2} \mathcal{L}_{j}^{\dagger} \mathcal{L}_{j} \rho\left(t^{\prime}\right)-\frac{1}{2} \rho\left(t^{\prime}\right) \mathcal{L}_{j}^{\dagger} \mathcal{L}_{j}\right)
$$

where $T_{j}$ represents the time constant of a particular dissipation process $j$. Time evolution of the density matrix $\rho\left(t^{\prime}\right)$ of the quantum system under a Hamiltonian $H\left(t^{\prime}\right)$ is treated in the Liouville-von Neumann formalism with the extension of dissipative and pure dephasing effects. In the case of cresyl violet, we assume that the density matrix elements $\rho=\sum_{m}^{M} \sum_{n}^{N} P_{m n}|m\rangle\langle n|$ with $m, n \in\left\{g, e_{\text {rel }}, e, e^{\prime}, f, f^{\prime}\right\}$ result from a six-level system, where the energies of $|e\rangle$ and $\left|e^{\prime}\right\rangle$ were determined from the linear absorption spectrum and the energy of $\left|e_{\text {rel }}\right\rangle$ is given by the energy of $|e\rangle$ minus half the Stokes shift energy. The energies $|f\rangle$ and $\left|f^{\prime}\right\rangle$ are based on the value of the energy shift $\Delta$, which was taken from the experimental 2Q 2D spectrum. We then conducted a series of simulations with varying energy shift $\Delta$ in order to find the optimum agreement of experimental and simulated data. For 
that purpose, we determined the root-mean-square deviation of the simulated data (real part of $2 \mathrm{Q}$ and $1 \mathrm{Q}-2 \mathrm{Q})$ with respect to the experimental data. $H\left(t^{\prime}\right)$ is expressed as the sum of a time-independent Hamiltonian $H_{0}=\hbar \omega_{m} \sum_{m}^{M}|m\rangle\langle m|$ and an interaction Hamiltonian $H_{I}\left(t^{\prime}\right)=\gamma_{e x} E(t) \sum_{m \neq n} \gamma_{m n}(|m\rangle\langle n|+| n\rangle\langle m|)$, where the overall coupling $\gamma_{e x}$ of the external field $E(t)$ was set to 0.015 . We set the individual relative transition strengths $\gamma_{m n}$ as $\gamma_{e g}=1.0$ and $\gamma_{e^{\prime} g}=0.72$ (determined from the linear absorption spectrum by taking the square root of normalized absorption at $\hbar \omega_{e g}=2.058 \mathrm{eV}$ and $\hbar \omega_{e^{\prime} g}=2.203 \mathrm{eV}$, respectively). Earlier measurements of cresyl violet have shown that there is non-negligible excited-state absorption (ESA) in the range of the utilized laser spectrum. ${ }^{50}$ The vastly different experimental parameters and response-function contributions make a quantitative comparison of the two methods difficult, though. We varied $\gamma_{e f}$ and $\gamma_{e^{\prime} f^{\prime}}$ systematically and find that $\gamma_{e f}=2.0$ and $\gamma_{e^{\prime} f^{\prime}}=2.0$ reproduce the peak amplitudes from our experimental findings best. The Lindblad operators $\mathcal{L}_{j}^{\dagger}$ and $\mathcal{L}_{j}$, which are $6 \times 6$ matrices, take the environmental effects on the system into account via dephasing and population relaxation. Here, a dephasing process is described by $\mathcal{L}_{j}^{d e p h}=|m\rangle\langle m|+(-1)| n\rangle\langle n|$. Population relaxation is given by $\mathcal{L}_{j}^{r e l}=|m\rangle\langle n|$ where only the matrix entries of the respective ket-bra are unity while all other entries are zero. The time constants $T_{j}$ characterizing pure dephasing and population relaxation processes between level pairs are utilized as parameters to manually fit the experimental peak amplitudes and lineshapes in the 2D spectra (see Supporting Information Section S3). The excitation laser field was calculated from the experimentally utilized laser spectrum (Figure 1d) and assuming a flat phase. A simulation applying a super-Gaussian fit (centered at $\hbar \omega_{0}=2.138 \mathrm{eV}$ ) of the measured laser spectrum as excitation field is provided for comparison in Figure S2 of Supporting Information, proving that the row-shaped features arise from the molecular response and not only from the somewhat modulated laser spectrum. 


\section{Supporting Information}

Discussion of fluorescence quantum yield influence on 2Q and 1Q-2Q 2D spectra, simulation with super-Gaussian fit of the laser spectrum, and simulation parameters.

\section{Acknowledgments}

European Research Council (ERC) Consolidator Grant "Multidimensional Ultrafast TimeInterferometric Spectroscopy of Coherent Phenomena in all Environments" (MULTISCOPE) and European Cooperation in Science and Technology (COST) Action MP1403 "Nanoscale Quantum Optics". We would like to thank Bernhard Huber for support with computations and further acknowledge valuable discussions with Sebastian Roeding, Jakub Dostál, and Christian Kramer.

\section{References}

(1) Jonas, D. M. Two-Dimensional Femtosecond Spectroscopy. Annu. Rev. Phys. Chem. 2003, 54, 425-463.

(2) Ogilvie, J. P.; Kubarych, K. J. Multidimensional Electronic and Vibrational Spectroscopy: An Ultrafast Probe of Molecular Relaxation and Reaction Dynamics. Adv. At. Mol. Opt. Phys. 2009, 57, 249-321.

(3) Cundiff, S. T.; Mukamel, S. Optical Multidimensional Coherent Spectroscopy. Phys. Today 2013, 66(7), 44-49.

(4) Cho, M. Two-Dimensional Optical Spectroscopy; CRC Press: Boca Raton, 2009.

(5) Wong, C. Y.; Scholes, G. D. Biexcitonic Fine Structure of CdSe Nanocrystals Probed by Polarization-Dependent Two-Dimensional Photon Echo Spectroscopy. J. Phys. Chem. A 2011, 115, 3797-3806. 
(6) Kuehn, W.; Reimann, K.; Woerner, M.; Elsaesser, T.; Hey, R. Two-Dimensional Terahertz Correlation Spectra of Electronic Excitations in Semiconductor Quantum Wells. J. Phys. Chem. B 2011, 115, 5448-5455.

(7) Ginsberg, N. S.; Cheng, Y.-C.; Fleming, G. R. Two-Dimensional Electronic Spectroscopy of Molecular Aggregates. Acc. Chem. Res. 2009, 42, 1352-1363.

(8) Nuernberger, P.; Ruetzel, S.; Brixner, T. Multidimensional Electronic Spectroscopy of Photochemical Reactions. Angew. Chem. Int. Ed. 2015, 54, 11368-11386.

(9) Bizimana, L. A.; Brazard, J.; Carbery, W. P.; Gellen, T.; Turner, D. B. Resolving Molecular Vibronic Structure Using High-Sensitivity Two-Dimensional Electronic Spectroscopy. J. Chem. Phys. 2015, 143, 164203.

(10) Marroux, H. J. B.; Orr-Ewing, A. J. Distinguishing Population and Coherence Transfer Pathways in a Metal Dicarbonyl Complex Using Pulse-Shaped Two-Dimensional Infrared Spectroscopy. J. Phys. Chem. B 2016, 120, 4125-4130.

(11) Hamm, P.; Lim, M.; DeGrado, W. F.; Hochstrasser, R. M. The Two-Dimensional IR Nonlinear Spectroscopy of a Cyclic Penta-Peptide in Relation to its Three-Dimensional Structure. Proc. Natl. Acad. Sci. U.S.A. 1999, 96, 2036-2041.

(12) Brixner, T.; Stenger, J.; Vaswani, H. M.; Cho, M.; Blankenship, R. E.; Fleming, G. R. Two-Dimensional Spectroscopy of Electronic Couplings in Photosynthesis. Nature 2005, 434, 625-628.

(13) Consani, C.; Auböck, G.; Mourik, F. v.; Chergui, M. Ultrafast Tryptophan-to-Heme Electron Transfer in Myoglobins Revealed by UV 2D Spectroscopy. Science 2013, 339, $1586-1589$.

(14) Lewis, N. H. C.; Fleming, G. R. Two-Dimensional Electronic-Vibrational Spectroscopy of Chlorophyll $a$ and b. J. Phys. Chem. Lett. 2016, 7, 831-837. 
(15) Ghosh, A.; Ostrander, J. S.; Zanni, M. T. Watching Proteins Wiggle: Mapping Structures with Two-Dimensional Infrared Spectroscopy. Chem. Rev. 2017, 117, 1072610759.

(16) Akhtar, P.; Zhang, C.; Do, T. N.; Garab, G.; Lambrev, P. H.; Tan, H.-S. TwoDimensional Spectroscopy of Chlorophyll a Excited-State Equilibration in LightHarvesting Complex II. J. Phys. Chem. Lett. 2017, 8, 257-263.

(17) Fulmer, E. C.; Mukherjee, P.; Krummel, A. T.; Zanni, M. T. A Pulse Sequence for Directly Measuring the Anharmonicities of Coupled Vibrations: Two-Quantum TwoDimensional Infrared Spectroscopy. J. Chem. Phys. 2004, 120, 8067-8078.

(18) Mukamel, S.; Oszwałdowski, R.; Yang, L. A Coherent Nonlinear Optical Signal Induced by Electron Correlations. J. Chem. Phys. 2007, 127, 221105.

(19) Kim, J.; Mukamel, S.; Scholes, G. D. Two-Dimensional Electronic Double-Quantum Coherence Spectroscopy. Acc. Chem. Res. 2009, 42, 1375-1384.

(20) Nemeth, A.; Sperling, J.; Hauer, J.; Kauffmann, H. F.; Milota, F. Compact PhaseStable Design for Single- and Double-Quantum Two-Dimensional Electronic Spectroscopy. Opt. Lett. 2009, 34, 3301-3303.

(21) Nemeth, A.; Milota, F.; Mančal, T.; Pullerits, T.; Sperling, J.; Hauer, J.; Kauffmann, H. F.; Christensson, N. Double-Quantum Two-Dimensional Electronic Spectroscopy of a Three-Level System: Experiments and Simulations. J. Chem. Phys. 2010, 133, 094505.

(22) Kim, J.; Huxter, V. M.; Curutchet, C.; Scholes, G. D. Measurement of Electron Electron Interactions and Correlations Using Two-Dimensional Electronic Double-Quantum Coherence Spectroscopy. J. Phys. Chem. A 2009, 113, 12122-12133. 
(23) Stone, K. W.; Gundogdu, K.; Turner, D. B.; Li, X.; Cundiff, S. T.; Nelson, K. A. Two-Quantum 2D FT Electronic Spectroscopy of Biexcitons in GaAs Quantum Wells. Science 2009, 324, 1169-1173.

(24) Stone, K. W.; Turner, D. B.; Gundogdu, K.; Cundiff, S. T.; Nelson, K. A. ExcitonExciton Correlations Revealed by Two-Quantum, Two-Dimensional Fourier Transform Optical Spectroscopy. Acc. Chem. Res. 2009, 42, 1452-1461.

(25) Christensson, N.; Milota, F.; Nemeth, A.; Pugliesi, I.; Riedle, E.; Sperling, J.; Pullerits, T.; Kauffmann, H. F.; Hauer, J. Electronic Double-Quantum Coherences and Their Impact on Ultrafast Spectroscopy: The Example of $\beta$-Carotene. J. Phys. Chem. Lett. 2010, 1, 3366-3370.

(26) Konold, P. E.; Jimenez, R. Excited State Electronic Landscape of mPlum Revealed by Two-Dimensional Double Quantum Coherence Spectroscopy. J. Phys. Chem. B 2015, $119,3414-3422$.

(27) Gellen, T. A.; Bizimana, L. A.; Carbery, W. P.; Breen, I.; Turner, D. B. Ultrabroadband Two-Quantum Two-Dimensional Electronic Spectroscopy. J. Chem. Phys. 2016, 145, 064201.

(28) Tian, P.; Keusters, D.; Suzaki, Y.; Warren, W. S. Femtosecond Phase-Coherent TwoDimensional Spectroscopy. Science 2003, 300, 1553-1555.

(29) Tekavec, P. F.; Lott, G. A.; Marcus, A. H. Fluorescence-Detected Two-Dimensional Electronic Coherence Spectroscopy by Acousto-Optic Phase Modulation. J. Chem. Phys. 2007, 127, 214307.

(30) Aeschlimann, M.; Brixner, T.; Fischer, A.; Kramer, C.; Melchior, P.; Pfeiffer, W.; Schneider, C.; Strüber, C.; Tuchscherer, P.; Voronine, D. V. Coherent Two-Dimensional Nanoscopy. Science 2011, 333, 1723-1726. 
(31) Lott, G. A.; Perdomo-Ortiz, A.; Utterback, J. K.; Widom, J. R.; Aspuru-Guzik, A.; Marcus, A. H. Conformation of Self-Assembled Porphyrin Dimers in Liposome Vesicles by Phase-Modulation 2D Fluorescence Spectroscopy. Proc. Natl. Acad. Sci. U.S.A. 2011, 108, 16521-16526.

(32) Perdomo-Ortiz, A.; Widom, J. R.; Lott, G. A.; Aspuru-Guzik, A.; Marcus, A. H. Conformation and Electronic Population Transfer in Membrane-Supported Self-Assembled Porphyrin Dimers by 2D Fluorescence Spectroscopy. J. Phys. Chem. B 2012, 116, 10757-10770.

(33) Nardin, G.; Autry, T. M.; Silverman, K. L.; Cundiff, S. T. Multidimensional Coherent Photocurrent Spectroscopy of a Semiconductor Nanostructure. Opt. Express 2013, 21, 28617-28627.

(34) Widom, J. R.; Johnson, N. P.; Hippel, P. H. v.; Marcus, A. H. Solution Conformation of 2-Aminopurine Dinucleotide Determined by Ultraviolet Two-Dimensional Fluorescence Spectroscopy. New J. Phys. 2013, 15, 025028.

(35) Widom, J. R.; Lee, W.; Perdomo-Ortiz, A.; Rappoport, D.; Molinski, T. F.; AspuruGuzik, A.; Marcus, A. H. Temperature-Dependent Conformations of a Membrane Supported Zinc Porphyrin Tweezer by 2D Fluorescence Spectroscopy. J. Phys. Chem. A 2013, 117, 6171-6184.

(36) Karki, K. J.; Widom, J. R.; Seibt, J.; Moody, I.; Lonergan, M. C.; Pullerits, T.; Marcus, A. H. Coherent Two-Dimensional Photocurrent Spectroscopy in a PbS Quantum Dot Photocell. Nat. Commun. 2014, 5, 5869.

(37) De, A. K.; Monahan, D.; Dawlaty, J. M.; Fleming, G. R. Two-Dimensional Fluorescence-Detected Coherent Spectroscopy with Absolute Phasing by Confocal Imaging of a Dynamic Grating and 27-Step Phase-Cycling. J. Chem. Phys. 2014, 140, 194201. 
(38) Draeger, S.; Roeding, S.; Brixner, T. Rapid-Scan Coherent 2D Fluorescence Spectroscopy. Opt. Express 2017, 25, 3259-3267.

(39) Joo, T.; Jia, Y.; Yu, J.-Y.; Lang, M. J.; Fleming, G. R. Third-Order Nonlinear Time Domain Probes of Solvation Dynamics. J. Chem. Phys. 1996, 104, 6089.

(40) Nagasawa, Y.; Watanabe, A.; Takikawa, H.; Okada, T. Solute Dependence of Three Pulse Photon Echo Peak Shift Measurements in Methanol Solution. J. Phys. Chem. A 2003, 10\%, 632-641.

(41) Bolzonello, L.; Polo, A.; Volpato, A.; Meneghin, E.; Cordaro, M.; Trapani, M.; Fortino, M.; Pedone, A.; Castriciano, M. A.; Collini, E. Two-Dimensional Electronic Spectroscopy Reveals Dynamics and Mechanisms of Solvent-Driven Inertial Relaxation in Polar BODIPY Dyes. J. Phys. Chem. Lett. 2018, 9, 1079-1085.

(42) Tan, H.-S. Theory and Phase-Cycling Scheme Selection Principles of Collinear Phase Coherent Multi-Dimensional Optical Spectroscopy. J. Chem. Phys. 2008, 129, 124501.

(43) Kasha, M. Characterization of Electronic Transitions in Complex Molecules. Discuss. Faraday Soc. 1950, 9, 14.

(44) Lindblad, G. On the Generators of Quantum Dynamical Semigroups. Commun. Math. Phys. 1976, 48, 119-130.

(45) Heisler, I. A.; Moca, R.; Camargo, F. V. A.; Meech, S. R. Two-Dimensional Electronic Spectroscopy Based on Conventional Optics and Fast Dual Chopper Data Acquisition. Rev. Sci. Instrum. 2014, 85, 063103.

(46) Perlík, V.; Hauer, J.; Šanda, F. Finite Pulse Effects in Single and Double Quantum Spectroscopies. J. Opt. Soc. Am. B 2017, 34, 430-439.

(47) Smallwood, C. L.; Autry, T. M.; Cundiff, S. T. Analytical Solutions to the Finite-Pulse 
Bloch Model for Multidimensional Coherent Spectroscopy. J. Opt. Soc. Am. B 2017, 34, 419 .

(48) Ma, X.; Dostál, J.; Brixner, T. Broadband 7-fs Diffractive-Optic-Based 2D Electronic Spectroscopy Using Hollow-Core Fiber Compression. Opt. Express 2016, 24, 2078120791.

(49) Amat-Roldán, I.; Cormack, I.; Loza-Alvarez, P.; Gualda, E.; Artigas, D. Ultrashort Pulse Characterisation with SHG Collinear-FROG. Opt. Express 2004, 12, 1169-1178.

(50) Nishiyama, K.; Asano, Y.; Hashimoto, N.; Okada, T. Solvation Dynamics of Dye Molecules in Polar Solvents Studied by Time Resolved Hole Burning Spectroscopy. J. Mol. Liq. 1995, 65-66, 41-48. 